\title{
Toints
}

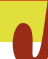

\section{Assessing hospital cost of joint arthroplasty}

\author{
FILIPPO BONIFORTI
}

UO Ortopedia e Traumatologia, Fondazione San Raffaele Giglio, Cefalù, Italy

\begin{abstract}
Purpose: total joint replacement is one of the most successful procedures in medicine and cost reimbursements to hospitals for the joint arthroplasty diagnosisrelated group are among the largest payments made by a Regional Health Service. Despite the popularity of these procedures, there are few high-quality costeffectiveness studies on this topic. This study evaluates the cost of total joint arthroplasty performed in a district hospital.

Methods: direct and indirect costs have been measured and patient procedure pathway was analyzed subdivided into three stages: surgical procedure, inpatient care and outpatient clinic.

Results: the cost of the surgical procedure stage was calculated as 3,798 euros, while that of the inpatient stage was 2,924 euros. The mean hospital costs per procedure amounted to 6,952 euros.

Conclusions: although the Health Service tariffs fully reimburse the cost of providing a joint replacement, our data contribute to point out the role of hospital staff's organization to support sustainable improvements on health care for joint replacement surgery.

Level of evidence: Level VI, single economic evaluation.
\end{abstract}

Keywords: joint, arthroplasty, finance, hospital, disease-related groups.

\section{Corresponding Author:}

Filippo Boniforti, MD

UO Ortopedia e Traumatologia, Fondazione

San Raffaele Giglio

Contrada Pietra Pollastra, 90015 Cefalù, (PA), Italy

E-mail: filippo.boniforti@gmail.com

\section{Introduction}

Arthroplasties for the management of hip and knee arthritis are highly successful procedures (1). Patients report pain relief and improvements in function after total joint arthroplasty (TJA) and the demand for TJA is expected to increased substantially in the coming years $(2,3)$. TJA is associated with costs to the health system and utilization rates have been found to differ between regions (4). Understanding the costs and value of care has become a focal issue for healthcare providers $(5,6)$. It has been suggested, in the Patient Protection and Affordable Care Act (USA, 2011), and by some economists, that cost-effectiveness analysis (CEA) may be an appropriate methodology for resource allocation $(7,8)$. CEA seeks to evaluate the economics of a healthcare intervention from the perspective not only of extending life, but also of improving its quality. However, financial analysis of a procedure has to be considered the first step, and the hypothesis investigated in the present study was that reimbursement by the public Regional Health Service (RHS) can cover the entire hospital cost of TJA in an Italian district hospital.

\section{Methods}

The costs and workload generated by primary joint replacement were evaluated at the Orthopedics and Trauma Department of the Fondazione San Raffaele Giglio Hospital (HSRG). The following parameters were measured in relation to yearly activity: personnel, instruments, operating theater, implants, management and financial costs. In detail, the gross costs of TJA (primary hip or knee replacement) were analyzed and classified with reference to the patient procedure pathway (PPP). The PPP comprises four stages: pre- 
admission clinic (PA), surgical procedure (SP), inpatient care (IP) and outpatient clinic (OP). Times, equipment and costs were measured for the entire pathway. The items taken into account in the different stages were the following:

PA - orthopedic surgeon and physician evaluations, chest X-ray and knee or hip joint X-ray examinations, blood and urine tests, consultation with an anesthetist, consent and clinical notes (any specialist consultations and diagnostic tests performed following the detection of co-morbidities were considered separately). SP - operating theatre sessions and surgical procedure, prosthesis or implant, anesthesiologist treatments and recovery.

IP - hospital admission, prophylactic drugs and chronic pharmaceutical treatments, postoperative period, start of rehabilitation program and hospital discharge. OP - clinical evaluation within two to five weeks of the surgery, X-ray within five weeks and prophylactic antithromboembolic drugs for four weeks after surgery.

In the financial analysis of the PPP, costs were measured in euros. The hospital pharmacy, which keeps a register of drugs and disposables, provided a list of all the equipment needed for TJA procedures. The hospital finance department's supply office indicated the prices of drugs and medical devices, while the costs for medical staff, nurses, administrative and technical personnel were measured by the hospital finance department's personnel office. Overall cost reports were produced by the hospital finance department. Direct and indirect costs of general hospital supplies were related to TJA procedures and analyzed for a 12 month period. The orthopedic department prospectively measured costs and surgical activity for TJA procedures over a three-month period. The results were evaluated in order to measure the average cost of one procedure within a hospital unit performing more than 300 TJAs per year. Reimbursement by the RHS for the code number 544, primary hip and knee joint replacement, during the analyzed period was 8861.77 euros per procedure.

\section{Results}

The costs for drugs and disposables during the three months of the study related to 86 primary procedures: 40 hips and 46 knees. The surgical procedures were performed by a single surgeon in a single operating theatre. All the patients were evaluated in the PA. The patients took an average of 3.7 hours in our outpatient clinic to complete the PA stage. All received orthopedic surgeon, anesthesiologist and nurse consultations. $\mathrm{X}$-rays and ECG examinations were performed and blood and urine samples were collected. No drugs were administered. Fifteen patients presented co-morbidities - vascular disease $(n=4)$, cardiac disease $(n=6)$, diabetes $(n=3)$ and respiratory disease $(n=2)-$ and required specific evaluations. The costs of $\mathrm{PA}$, relating to clinical evaluations, note taking, tests and indirect costs, were evaluated to be 115 euros per patient. Patients were admitted to hospital in the afternoon of the day before the operation and prepared for surgery. The SP stage began the morning of the operating theatre session and was measured from the patient's arrival in theatre to the time he/she was sent back to the ward. Patients were prepared with antibiotic prophylaxis, vein lines and urinary catheters. Epidural anesthesia was administered to all the patients. Surgery was performed by an experienced surgeon and it took from 45 to 115 minutes, skin to skin, with an average time per surgical procedure of 75 minutes. In all cases, the patients were sent back to the ward, awake, within 45 minutes of the end of the operation. The SP stage took from 120 to 210 minutes (average:140 minutes). Wraps, gowns and equipment were packed in a single kit containing instruments regularly used by the surgical team: this kit cost 35 euros per procedure. The cost of drugs, needles, catheters, drains, fluids and the patient's gown was calculated as 27 euros, while the cost of bone cement, surgical lavage, power bone saw and surgical drains was calculated as 185 euros. The surgical team comprised two surgeons, an anesthesiologist, scrub and runner nurses and an anesthesiologist nurse. The cost of the operating theatre sessions was analyzed by the finance and personnel departments of the HSRG and measured as: 880 euros per hour of surgical procedure and 450 euros for the admission and recovery stages. The cost of the prosthesis was calculated as 1,850 euros for the knee, and 2,175 euros for the hip. The average price of implants, calculated considering the 86 implants evaluated in this study, was 2,001 euros per implanted prosthesis. The gross costs of the SP stage were calculated as 3,798 euros per patient. The aspects taken into account when evaluating the IP stage were clinical assessments, fluids 
and drugs. One unit of blood was transfused in 11 cases. An average of three blood tests per patient, two ECGs, one X-ray of the operated joint, two surgical site dressings, three changes of bed sheets and 18 meals were provided during the IP stage, which lasted an average of six days. Overall costs for 86 patients have been measured in 3,341 euros for pharmacy (drugs and disposables), with an average of 38.84 euros per person. Meals have been measured with an average of 113 euros for each patient; average logistic and administrative costs were 27.68 euros and average laboratory tests costs were 107.02 euros per person. Overall costs supported by the hospital for physicians, nurses, health personnel and physical therapy have been measured to be 226,722 euros (average, 2,637 per patient). The cost IP stage was calculated as 2,924 euro per patient. The OP stage at 2 and 5 weeks after surgery for removal of stitches, clinical evaluations and joint $\mathrm{X}$-ray at 5 weeks, takes 15 minutes per patient per follow-up visit and anti-thromboembolic prophylaxis drugs for 5 weeks were calculated to have an average cost of 115 euros per patient.

The overall cost of TJA procedure at HSRG was estimated to be 6,952 euros, in front of 8861.77 euro of reimbursement. The SP was found to account for more than $55 \%$ of all the costs (Fig. 1). Personnel accounts for more than $50 \%$ of the total amount (Fig. 2). Equipment and instruments cost 2,650 euros and the prosthesis accounting for about $70 \%$ of the all lot (Fig. 3). The cost of personnel was found to be 4,297 euros (Fig. 4).

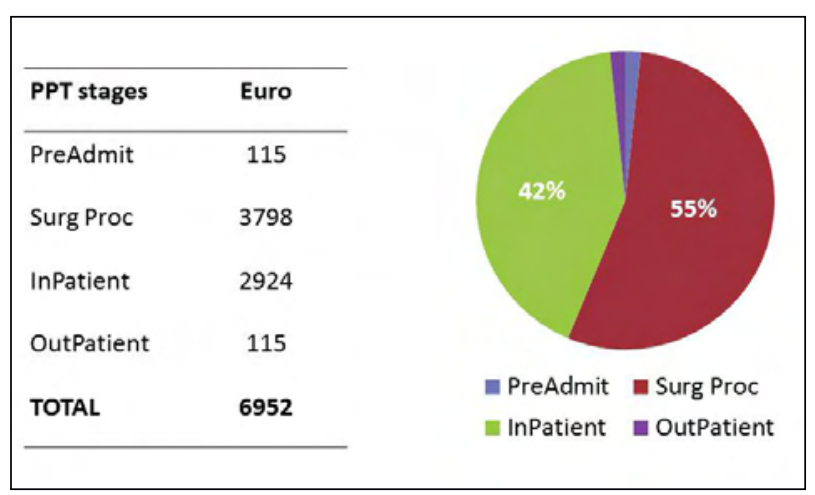

Fig. 1. Costs of the different stages in the patient procedure pathway in euros. The surgical procedure accounts for more than $50 \%$ of the costs of the entire joint arthroplasty pathway.

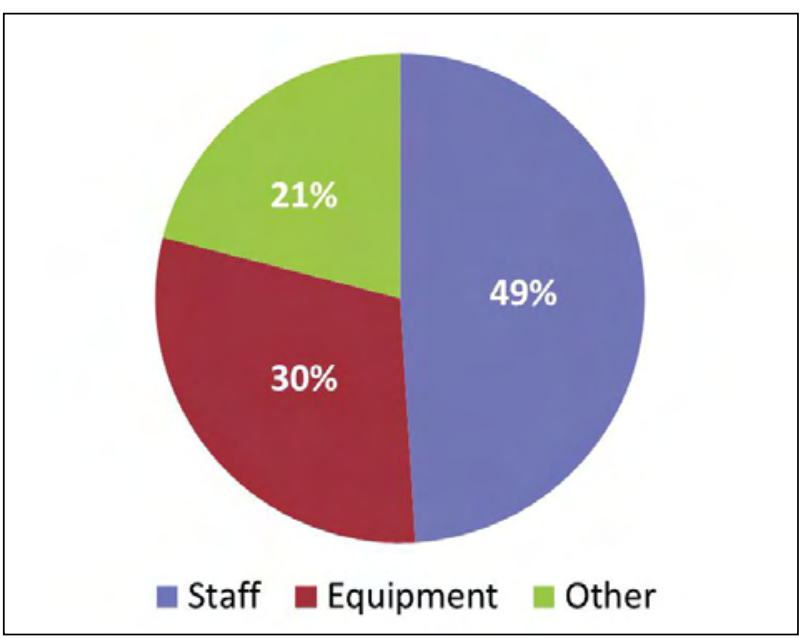

Fig. 2. Costs of reimbursement for staff, equipment and others as percentages of the cost of the joint arthroplasty procedure. Personnel accounts for near than $50 \%$ of the total amount.

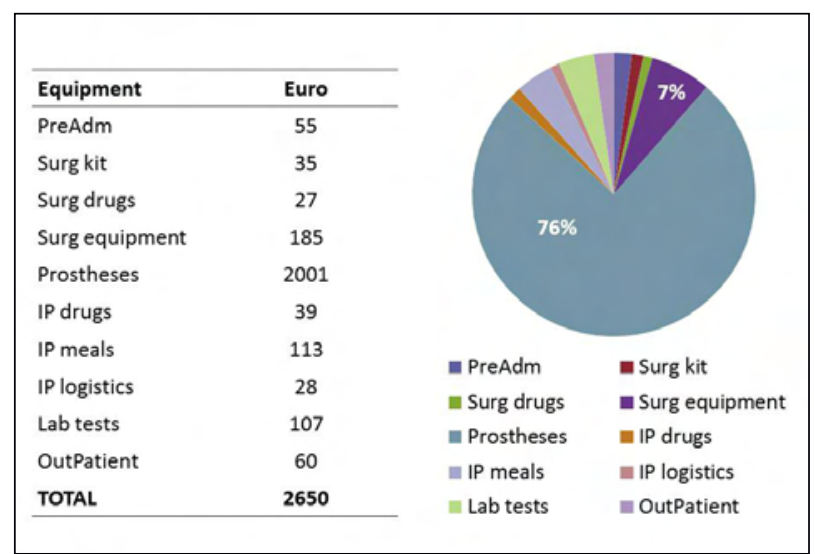

Fig. 3. Cost of equipment and instruments in euros for one patient procedure pathway. The cost of the implant accounts for $76 \%$ of the total amount.

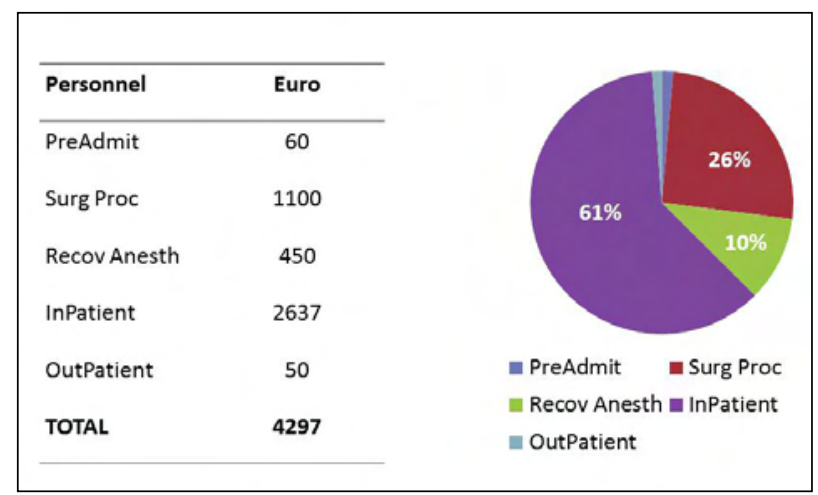

Fig. 4. Cost of staff and personnel in euros relating to the workload for one patient procedure pathway. The inpatient stage accounts for nearly $2 / 3$ of the entire cost of staffing. 


\section{Discussion}

In the context of measuring the value of clinical pathways for TJA, costs should be considered across the full continuum of care, from patient referral to an orthopedic surgeon through surgery, recovery, and rehabilitation which takes nearly 12 weeks (9). The costs we have analyzed rely on the 6 weeks after surgery. Bumpass et al. (10) reported QUALY measurement in hip and knee replacement patients by capturing all the meaningful differences in costs and outcomes between the intervention and comparators and described the procedure as of great value for patients and healthcare system.

In the study by Pasdera (11) for the Società Italiana Ortopedia e Traumatologia, costs related to TJA were reported to be significantly higher than those found in our study. However, health resources should be tracked in detail and discrepancies in cost assessments should be assessed according to the differences in the local healthcare systems. Readers should be aware that a limitation of our study is that we considered only two clinical pathways: primary total hip and primary total knee replacement.

We have illustrated some of the key principles for the economic evaluation of the surgical orthopedic interventions and we have captured costs from the surgeons viewpoint. Ideally, all elements of the care process should be tracked, but this is not always practical in a public health system given the array of elements in the care process and the complex system of care delivery. Moreover, it should be underlined the significant role of the staff and related activities in the saving cost management plan (12). The length of hospital stay, a parameter commonly used to measure hospital performances and financial effort, may change costs of the procedure for $7 \%$ per day. It can makes differences among quality of care.

The price of TJA prostheses has remained substantially stable over the last decade and manufacturers gain market shares through design and innovation. Faulkner et al. created a cost-effectiveness model for the British National Health Service to justify increased implant costs (13). They found implant cost, hospital cost and revision rate to be the main variables determining costs, and they underlined that a theoretical new hip prosthesis with $0 \%$ revision rate should cost no more than twice the cost of a cemented Charnley hip to maintain equivalent cost effectiveness. Gillespie et al. fixed the guideline for assessing the new implants cost (14). Development of National Registries and the Registro Italiano Artroprotesi (RIAP) support the standardization of implant selection (15). Our RHS provides more than 12000 implants per year and if purchases operate in equipment as a network, rather than single hospital needed, further saving are possible. Our results showed the average gross cost of the implant to be 2001 euros, an amount which represents the $22 \%$ of the entire reimbursement. With the continuing economic downturn and health budgets being cut, more effort are needed to compensate the need of innovation and saving should contain the new pressure on already strained finances (16).

In examining costs along the continuum of care, it is important to differentiate between variability in resource use that occurs because of patient variables or patient outcomes. This would suggest that a standardized clinical pathway can reduce costs overall. Even if financial analysis reveals only minor differences between hospitals, on a larger scale it can save a lot of money. Our results highlighted the considerable economic resources needed for personnel involved in the PPP. Consequently, initiatives such as fast-track surgery, enhanced rehabilitation programs, multidisciplinary clinical pathways and standardized clinical care allow hospital cost reductions of nearly $20 \%(17,18)$. The hospital volume is also a significant parameter and the break point to be efficient could be fixed at 100 implant per year $(19,20)$. The fee-for-service payment model has increased utilization of services and healthcare stakeholders should focus on the value of the procedure and payment for care modifications should be considered $(21,22)$.

In conclusion, inpatient resources are the most important cost item in relation to a TJA, representing nearly the $60 \%$ of the entire reimbursement. The cost of the implant affects the QUALY of the procedure.

In order to cut this cost, RHSs should consider purchasing in network rather than individual hospital or single orthopedic surgeons. High-quality prospective data are essential to drive resource allocation and robust methodologies should be used to improve the PPP. 


\section{oints}

\section{References}

1. Ethgen $\mathrm{O}$, Bruyere $\mathrm{O}$, Richy $\mathrm{F}$, et al. Health-related quality of life in total hip and total knee arthroplasty. A qualitative and systematic review of the literature. J Bone Joint Surg Am. 2004;86:963-974.

2. Day JS, Lau E, Ong KL, et al. Prevalence and projections of total shoulder and elbow arthroplasty in the United States to 2015. J Shoulder Elbow Surg. 2010;19:1115-1120.

3. Kurtz S, Ong K, Lau E, et al. Projections of primary and revision hip and knee arthroplasty in the United States from 2005 to 2030. J Bone Joint Surg Am. 2007;89:780-785.

4. Fisher ES, Bell JE, Tomek IM, et al. Trends and Regional Variation in Hip, Knee, and Shoulder Replacement. 2010. Available at: http://www.dartmouthatlas.org/downloads/ reports/Joint_Replacement_0410.pdf.

5. Cutler D, Wikler E, Basch P. Reducing administrative costs and improving the health care system. $\mathrm{N}$ Engl J Med. 2012;367:1875-1878.

6. Lansky D, Nwachukwu BU, Bozic KJ. Using financial incentives to improve value in orthopaedics. Clin Orthop Relat Res. 2012;470:1027-1037.

7. Schwartz JA, Pearson SD. Cost consideration in the clinical guidance documents of physician specialty societies in the United States. JAMA Intern Med. 2013;173:1091-1097.

8. Neumann PJ, Rosen AB, Weinstein MC. Medicare and costeffectiveness analysis. N Engl J Med. 2005;353:1516-1522.

9. Bozic KJ, Saleh KJ, Rosenberg AG, et al. Economic evaluation in total hip arthroplasty: analysis and review of the literature. J Arthroplasty. 2004;19:180-189.

10. Bumpass DB, Nunlay RM. Assessing the value of a total joint replacement. Curr Rev Musculoskelet Med. 2012;5:274-282.

11. Pasdera A. I costi per DRG: i risultati della ricerca della Società Italiana di Ortopedia e Traumatologia (S.I.O.T.). GIOT. 2008;2:44-52.

12. Rana AJ, Iorio R, Healy WL. Hospital economics of primary
THA decreasing reinbursement and increasing cost, 1990 to 2008. Clin Orthop Relat Res. 2011;469:355-361.

13. Faulkner A, Kennedy LG, Baxter K, et al. Effectivness of hip prostheses in primary total hip replacement: a critical review of evidence and an economic model. Health Tecnol Assess. 1998;2:1-133.

14. Gillespie WJ, Pekarsky B, O'Connell DL. Evaluation of new technologies for total hip replacement. Economic modelling and clinical trials. J Bone Joint Surg Br. 1995;77:528-533.

15. Luzi I, Del Manso M, Romanini E, et al. Interventi di artroprotesi: analisi dati SDO. RIAP primo report. Il Pensiero Scientifico Editore. 2014;90-117.

16. Iorio R, Davis CM, Healy WL, et al. Impact of the economic downturn on adult reconstruction surgery: a survey of the American Association of Hip and Knee Surgeons. J Arthroplasty. 2010,25:1005-1014.

17. Healy WL, Iorio R, Ko J, et al. Impact of cost reduction programs on short-term patient outcome and hospital cost of total knee arthroplasty. J Bone Joint Surg Am. 2002;84-A:348353.

18. Marshall DA, Wasylak T, Khong $\mathrm{H}$, et al. Measuring the value of total hip and knee arthroplasty: considering costs over the continuum of care. Clin Orthop Rel Res. 2012;470:10651072.

19. Martineau P, Filion KB, Huk OL, et al. Primary hip arthroplasty costs are greater in low-volume than in high-volume Canadian hospitals. Clin Orthop Relat Res. 2005,XX:152-156.

20. Kallala RF, Vanhegan IS, Ibrahim MS, et al. Financial analysis of revision knee surgery based on NHS tariffs and hospital costs. does it pay to provide a revision service? Bone Joint J Br. 2015,97-B:197-201.

21. Cutler D, Wikler E, Basch P. Reducing administrative costs and improving the health care system. $N$ Engl J Med. 2012;367:1875-1878

22. Rana AJ, Bozic KJ. Bundled payments in orthopedics. Clin Orthop Relat Res. 2015;473:422-425. 